\title{
Emodin: Its role in prostate cancer-associated inflammation (Review)
}

\author{
YANJIE TU ${ }^{1,2}$, ZHONGHUA WU ${ }^{1,3}$, BO TAN ${ }^{1,2,4}$, AIDONG YANG ${ }^{1,2}$ and ZHAOQIN FANG ${ }^{1,3}$ \\ ${ }^{1}$ Research Center for The Application of Classical Prescriptions; ${ }^{2}$ Department of Febrile Disease, Basic Medical College; \\ ${ }^{3}$ Department of Experimental TCM, ${ }^{4}$ Shuguang Hospital, Shanghai University of \\ Traditional Chinese Medicine, Shanghai 201203, P.R. China
}

Received March 12, 2019; Accepted July 4, 2019

DOI: 10.3892/or.2019.7264

\begin{abstract}
Prostate cancer is one of the most common malignancies diagnosed in males. Cancer-related inflammatory factors include tumor necrosis factor, inflammasomes, cytokines, chemokines, transcription factors, infiltrating or circulating immune cells, reactive oxygen species, and sex hormone receptors. These are mainly associated with the local immune response at the tumor site. Emodin, a chemical compound that can be isolated from the plant rhubarb among others, has been shown to exhibit anti-inflammatory and anticancer properties in prostate cancer. This review summarizes the effects of emodin on prostate cancer and analyzes whether it interferes with prostate cancer through anti-inflammatory pathways. New information regarding the development of emodin derivatives including their increased solubility and reduced side effects through chemical structure modifications is also reviewed.
\end{abstract}

Correspondence to: Dr Aidong Yang or Dr Zhaoqin Fang, Research Center for The Application of Classical Prescriptions, Basic Medical College, Shanghai University of Traditional Chinese Medicine, 1200 Cailun Road, Pudong New District, Shanghai 201203, P.R. China E-mail: aidongy@126.com

E-mail: zqfang@sh163.net

Abbreviations: PIA, proliferative inflammatory atrophy; PIN, prostatic intraepithelial neoplasia; $\mathrm{CZ}$, central zone; TZ, transition zone; PZ, peripheral zone; BPH, benign prostatic hyperplasia; HIF1, hypoxia-inducible factor 1; PSA, prostate-specific antigen; TAMs, tumor-associated macrophages; EMT, epithelial-mesenchymal transdifferentiation; CTL, cytotoxic T lymphocytes; NK, natural killer; ROS, reactive oxygen species; RNS, reactive nitrogen species; SNP, single nucleotide polymorphism; AR, androgen receptor; VEGF, vascular endothelial growth factor; MDR, multidrug resistance; HSP90, heat shock protein 90; SARMs, selective androgen receptor modulators; PD-L1, programmed death ligand 1; PD-1, programmed death protein 1; MSCs, mesenchymal stem cells; miRNAs, microRNAs; DNAm, DNA methylation; mTORC, mammalian target of rapamycin complex; PTEN, phosphatase and tensin homolog

Key words: prostate cancer, emodin, inflammation, immune therapy

\section{Contents}

1. Introduction

2. Inflammation in prostate cancer development

3. Antitumor effect of emodin in prostate cancer

4. Antitumor effects of emodin derivatives in prostate cancers

5. Emodin analog development in prostate cancer

6. Prospects of emodin in the inflammatory immune microenvironment of prostate cancer

\section{Introduction}

Prostate cancer is one of the most common malignancies diagnosed in males (1). The pathogenesis of prostate cancer has not been clearly elucidated to date, and requires further exploration to facilitate early diagnosis and effective treatment (2). Prostate is a human organ that is easily susceptible to infections and inflammations. Chronic inflammation plays a significant role in the development of various types of cancer including prostate cancer (3).

Approximately $15-20 \%$ of cancer patients are associated with infections or inflammation and prostate cancer is one such cancer type (4). In a self-reported prospective cohort study of 5,821 men over 65 years of age, chronic prostatitis was shown to be a significant factor in the occurrence of prostate cancer (5). Chronic inflammation triggers proliferative inflammatory atrophy (PIA) of the prostate, which in turn acts as a potential precursor lesion to prostatic intraepithelial neoplasia (PIN) and carcinoma (6).

Emodin (1,3,8-3 hydroxy-6-methyl anthraquinone), with a molecular formula of $\mathrm{C}_{15} \mathrm{H}_{10} \mathrm{O}_{5}$, (Fig. 2A) is an active ingredient of the plant rhubarb, and is mostly used for relieving abdominal distension, constipation and other gastrointestinal symptoms (7). Recent studies show that emodin exhibits anti-inflammatory and anticancer effects on prostate cancer (8). In this review, we aim to clarify the correlation between inflammation and prostate cancer, as well as the mechanism of action of emodin in prostate cancer to explore its role in the inhibition of prostate cancer through anti-inflammatory pathways.

\section{Inflammation in prostate cancer development}

Correlation between inflammation and prostate cancer. Inflammation is regarded as a hallmark of the occurrence and 
development of cancer (9). In 1863, Rudolf Virchow found leukocytes in neoplastic tissues and hypothesized 'lymphatic infiltration' as the first step in cancer progression in chronic inflammatory regions. In the 1990s, the study of an inflammatory microenvironment in tumor tissues was the main research focus in the field of cancer research (4). A sustained inflammatory microenvironment induces several reactive oxygen species (ROS), reactive nitrogen species (RNS), cytokines and chemokines, growth factors and other inflammatory mediators, changing the stability of the cellular environment. This in turn induces cell proliferation, chemotaxis, and inflammatory cells, leading to DNA oxidative damage. This indicates that the proliferation of these cells loose their control in the inflammatory microenvironment (10). This finally induces tumors, causing inflammation in the local environment of the tumor. Inflammation plays two roles in the turmor microenvironment. Firstly, it shows benign local effects, such as T-cell mediated cytotoxicity, leading to tumor inhibition; and secondly, the inflammation is characterized by persistent, adverse effects, such as promotion of malignant cell proliferation, angiogenesis, and metastasis, which subsequently destroys the immune response and weakens the body's response to hormones and chemotherapy drugs. This results in the occurrence of lymphoma B symptoms (i.e., fever, sweating, weight loss) and cachexia. At the same time, oncogenes can induce the formation of an inflammatory microenvironment (9) (Fig. 1).

The main signaling pathways, inflammatory mediators, inflammatory cells, and cytokines involved in the inflammatory immune system of prostate cancer. The prostate consists of three different regions: the central zone (CZ), the transition zone (TZ) and the peripheral zone (PZ). Benign prostatic hyperplasia (BPH) occurs in the $\mathrm{TZ}$ area, whereas prostatitis and cancer occur mainly in the PZ area (11). Several studies have reported that inflammatory mediators such as inflammatory pathways, inflammatory cytokines, and inflammatory cells promote the development and progression of prostate cancer (9). Both external inflammatory pathways and inherent genetic pathways can lead to activation of inflammatory signaling pathways, mainly nuclear factor (NF)- $\mathrm{KB}$ signaling, JAK/Stat, hypoxia-inducible factor 1 (HIF-1) signaling and mechanistic target of rapamycin (mTOR) signaling pathways (9). NF- $\kappa B$ is a crucial transcription factor in the inflammatory reaction, which increases the expression of tumor-promoting factors, such as interleukin (IL)- 6 and tumor necrosis factor (TNF) $-\alpha$. The crosstalk between NF- $\kappa B$ and multiple pathways, including Stat3, AP1, interferon regulatory factor, Nrf2, Notch, Wnt/ $\beta$-catenin affects cancer cell behaviors in regards to metabolism, invasion, metastasis, angiogenesis and resistance to treatment. The JAK/Stat pathway is an inflammatory pathway that is activated under moderate stress conditions. It is closely related with the occurrence and development of prostate cancer (12). Stat1 regulates immune suppression induced by macrophages and bone marrow-derived suppressor cells (BMSCs) through the expression of nitric oxide synthase. Stat3 is upregulated in cancer and immune cells, and is associated with increased synthesis of key inflammatory mediators, cytokines and chemokines. HIF1 signal transduction pathways are activated by oncogenes, which in turn can assist in identifying the hypoxic state of the tumor cells and adjust tumor metabolism by increasing glycolysis and reducing mitochondrial function to promote the survival of tumor cells (13). One reason for age-related cancers such as prostate cancer could be an inflammatory milieu driven by mTOR in senescent cells (14). The main function of TORC2 is phosphorylation of Akt at Ser473 and TORC2 was found to play an important role in the proliferation and anchorage-independent growth of PC-3 prostate cells. Akt was fount to be highly activated by phosphorylation at Ser473 in prostate cancer cell lines (PC-3 and LN-CaP). Knockdown of Rictor (a component of TORC2) reduced Akt Ser473, delaying prostate cancer cell proliferation (15).

All inflammatory cytokines (IL-1, IL-6, IL-11, IL-18, IL-7, TNF- $\alpha$ and mic-1/GDF-15) are involved in the occurrence of prostate cancer (11). Both IL-1 $\beta$ and TNF- $\alpha$ enhance the recruitment of immunosuppressive cells, increase the expression of chemokine factors on the tumor cell surface, promote the ability of tumor cell invasion and metastasis ability and finally promote tumor formation (16). IL-6 is an important inflammatory cytokine in the JAK/Stat pathway and a key regulatory factor in tumor formation (17). Previous studies revealed the existence of IL-6 in the epithelial cells of prostate cancer patients (18). A variety of inflammatory lesions stimulate IL-6, and together with its gp130 (GP130) subunit activates JAK/STAT, ERK1/2, MARK, PI3K, AKT and mTORC1 signaling pathways to regulate prostate cancer cell proliferation and apoptosis (18). The high expression of IL-17 in PIA lesions is the direct evidence for the involvement of the inflammatory microenvironment in the development of prostate cancer. The IL-17-MMP7 signaling pathway has been found to be involved in the transition of prostate epithelial neoplasia to prostate cancer (19). IL-18 helps in maintaining the inflammatory microenvironment of prostate cancer by damaging the function of natural killer (NK) cells and allowing tumor cells to escape the host immune response (13). The expression of IL-32 in the prostate gland helps reduce the occurrence of prostate cancer, and thus has become a 'hot' research topic in the treatment of prostate cancer (20). MIC-1/GDF-15 is regulated by inflammatory cytokines, and the combination of MIC-1/GDF-15 and prostate-specific antigen (PSA) in the serum can improve the specificity of prostate cancer detection (11). Tumor cells and inflammatory cells secrete TGF- $\beta$, which enhances the expression of CXCL12/CXCR4 and CXC5/CXCR2 in tumor cells, suggesting the initiation of tumor metastasis. INF- $\gamma$ plays a positive role in inhibiting tumor occurrence. Macrophage migration inhibitory factor (MIF) represents a link between inflammation and cancer. This promotes macrophages present in inflammation, local invasion, proliferation, activation and secretion of TNF- $\alpha$, IL-1 and IL-8 cytokines; and induces macrophages to produce NO, increasing DNA damage. Moreover, MIF with the help of Erk1/Erk2 phosphorylation events can activate NF- $\mathrm{kB}, \mathrm{COX}-2$ and increase NOS2, with or without dependence on P53 apoptosis inhibition (21). A new inflammatory factor (composed of NLR proteins) has recently been reported to be associated with prostate cancer cell apoptosis and can lead to the secretion and maturity of IL-1 and IL-18 cytokines (20).

In the tumor tissues of prostate cancer, distribution of tumor-associated macrophages (TAMs) can have an effect 

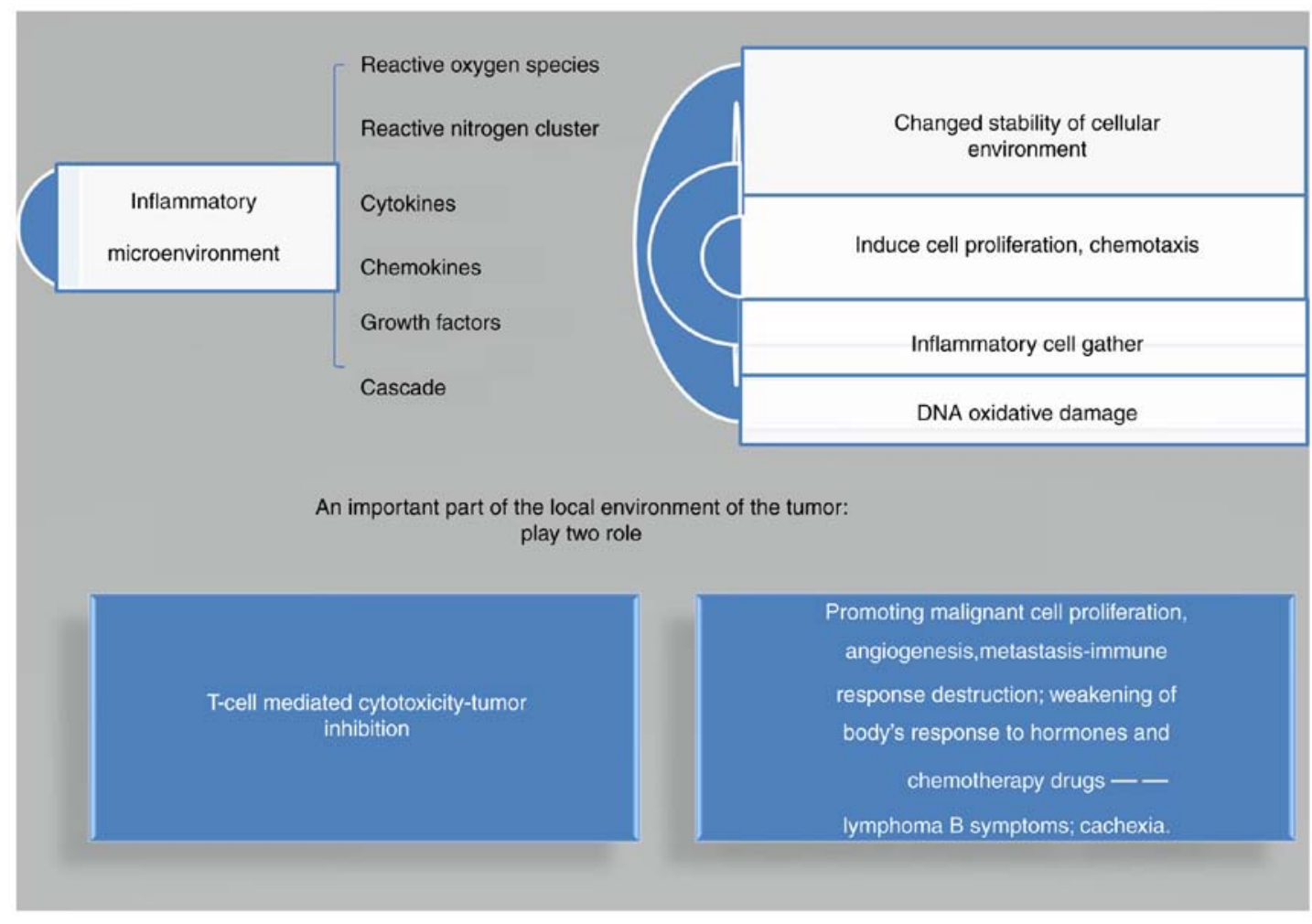

Figure 1. Correlation between inflammation and cancer.

on tumor stage and progression (21). The proportion of $\mathrm{CD}^{+}$ $\mathrm{T}$ cells in prostate cancer tissues was found to be much higher than that in peripheral blood, especially $\mathrm{TH}_{1}, \mathrm{TH}_{17}$ and Treg cells $(22,23)$. Infiltrating macrophages, mast cells, neutrophils, T cells, B cells and their related subgroups in the tumor release TNF- $\alpha$, epidermal growth factor (EGF), vascular endothelial growth factor (VEGF), fibroblast growth factor 2 (FGF2) and other cytokines. Infiltration of these inflammatory mediators could also mediate epithelial-mesenchymal transition (EMT), promoting tumor growth and metastasis. Furthermore, inflammation could induce differentiation of BMSCs and tumor stem cells into epithelial and interstitial fibroblast cells, thus promoting tumor growth and angiogenesis. Meanwhile, the inhibitory effects of BMSCs on cytotoxic T lymphocytes (CTLs) and NK cells also maintain tumor cell viability in the immune mechanism (24).

Other factors associated with inflammation and prostate cancer. Mitochondria, complement activation, ROS and RNS, DNA methylation, chemokines, innate immune genes, estrogen and oxidative stress are all also involved in the immunoregulatory function of prostate cancer. Mitochondria are known as the heart of immunity, and can activate main innate immune signaling pathways such as NF- $\mathrm{B}$ (25). In addition to the components of innate immunity, complement activation also participates in the adaptive immune response and inflammation. Complement activation end product and its receptor reduces cell apoptosis, promotes cell differentiation, proliferation, and migration by regulating immune response in the tumor microenvironment (26). Continuous generation of $\mathrm{ROS}\left(\mathrm{OH}^{-}, \mathrm{O}_{2}\right)$ and RNS (NO, $\mathrm{OONO}^{-}$) in chronic inflammation induces damage of the cellular macromolecules, especially DNA chain rupture and base mutation. This subsequently leads to tumor-suppressor gene mutation and protein modification after translation that is related to basic processes such as cell apoptosis, DNA repair, and cell cycle checkpoint, increasing the risk of chronic inflammation. In addition, DNA methylation is one of the major epigenetic changes, in which significant hypermethylation occurs in various tumors. The hypermethylation of the promoter can induce transcriptional silencing of APC, p16, BRCA1, Rb, MDM2, and other tumor-suppressor genes. The methylated $\mathrm{CpG}$ site aids in easily removing ammonia, leading to missense mutation of the tumor-related genes. Research has revealed that hypermethylation is often triggered by chronic inflammation in microbial infections, and thus DNA methylation also reflects the association between inflammation and cancer (10). Chemokines induce inflammatory cells towards the inflammatory site and surrounding lesions, regulating the occurrence, development, adhesion, and spread of prostate cancer cells (27). The migration and invasion ability of PC-3 cells were significantly improved after treatment with exogenous CXCL16, suggesting that CXCL16/CXCR6 might act as an independent chemokine axis in prostate bone metastasis (27). The expression of CXCL1/GRO $\alpha$ was found to be increased in prostate cancer after castration, promoting prostate cancer cell proliferation, migration and invasion by decreasing fibulin-1 expression through $\mathrm{NF}-\kappa \mathrm{B} / \mathrm{HDAC} 1$ epigenetic regulation (28). CXCR4 interacts with matrix proteins (such as laminin, fibronectin, collagen) in prostate cancer cells and acts on tumor cells through its ligand CXCL12, thus regulating the expression of synthase, FAK phosphorylation, p38MARK and ROCK kinase $(27,29)$. 
Two innate immune-related genes, the inactivated mutants E265X and M1I in L- ribonuclease (RNaseL), have been associated with prostate cancer susceptibility. RNase L, which is activated and expressed after a novel $\gamma$-retrovirus infection, is prone to cause prostatitis. RNase $\mathrm{L}$ when activated by its cognate-induced ligand induces 2',5'-related oligoadenylation, which is encoded by the MIC1 gene. Locus is defined as the site of susceptibility to prostate cancer. Single nucleotide polymorphisms (SNPs) in RNase L are implicated in inflammation and prostate cancer risk $(30,31)$.

Androgen receptors (ARs) are ligand-activated transcription factors of the nuclear receptor superfamily that mediate the biological effects of androgens in the prostate. AR signaling regulates inflammation, which in turn affects the progression of BPH and plays an important role in prostate cancer development and progression (32). Studies on immune inflammation conducted in $105 \mathrm{BPH}$ specimens showed that patients with stronger immune inflammation have larger prostate size, higher AR expression levels and higher serum PSA levels (33). Immunohistochemical analysis showed that BPH patients had more infiltrated macrophages in the prostate and higher expression of CCL3 than those with normal prostate. Stromal AR could increase the expression of CCL3 by recruiting infiltrating macrophages, which thereby promotes the development of BPH (34). Interestingly, the opposite effect of androgen/AR signaling showed that dihydrotestosterone can regulate the immune system in BPH by inhibiting inflammatory cytokines in stromal cells (35).

The overexpression of aromatase $\left(\mathrm{AROM}^{+}\right)$can increase mast cells in the prostatic tissues of mice affected by endogenous estrogen, and a large number of infiltrated inflammatory cells in the matrix and cavity of the prostate. According to PCR array, CCL20, CCL8, CCR6, CCR5, and CCR2 showed significant expression as bridge factors in the association of inflammation and cancer. The atypical epithelial cells and micropapillary growth pattern then appeared in the inflammatory infiltration area. Additionally, several atypical nuclei with prominent nucleoli appeared, and these changes were associated with high levels of stromal tumor cell proliferation in the surrounding tissues, suggesting chronic inflammation and precancerous lesions. Therefore, it is believed that estrogen acts as a connection linking chronic prostatitis and prostatic intraepithelial neoplasia (PIN) (36). Another potential mechanism in which inflammation is related with cancer is that the oxidative stress induced by inflammatory cytokines can lead to epigenetic recruitment in the sites of DNA injury, producing DNA methyltransferase, chromatin remodeling, and inhibiting factor complex. Therefore, a wide abnormal DNA methylation and transcriptional silencing of gene promoters occurred in prostate cancer progression and metastasis $(22,37)$. Oxidative stress can also make inflammatory cytokines, such as TNF release signals, leading to DNA cracking with proximity induction of AR signals, making prostate epithelial cells and TMPRSS2-ERG gene fusion, and promoting prostate cancer (38) (Table I).

\section{Antitumor effect of emodin in prostate cancer}

PIM1, a proto-oncogene, is responsible for encoding serine/threonine PIM1 kinase, and plays a vital role in regulating prostate cancer cell cycle and apoptosis. As a kinase inhibitor, emodin was found to selectively inhibit PIM kinase and inhibit the growth of DU-145 prostate cancer cells, which were isolated from the brain metastases of prostate cancer. DU145 cells are androgen-independent prostate cancer cells with low differentiation degree, and lack the expression of endogenous AR (39). PC 3 cells, isolated from the bone metastases of human prostate cancer, are low differentiated androgen-independent prostate cancer cells, without endogenous AR. PC3 prostate cancer cell experiments have shown that emodin inhibited the growth of PC3 cells by activating the Notch signaling pathway, inducing cell apoptosis, and blocking the cells in the G2/M phase. In addition, emodin inhibited the role of VEGF in anticancer mechanisms (40). Emodin was found to act as a strong growth inhibitor in LNCaP cells isolated from the left supraclavicular lymph node of a male patient in 1977 , which is sensitive to androgen, and the cytotoxic mechanism was related to the production of ROS. In oxygen and low-oxygen environment, emodin can reduce the expression of AR in LNCaP cells (41).

The chemokine receptor CXCR4-CXCL12 axis promotes invasion and metastasis of protate tumor cells. Emodin was found to inhibit the activation of $\mathrm{NF}-\kappa \mathrm{B}$ and to lower CXCR4 and HER2 at the transcription level, thereby inhibiting the invasion and metastasis of DU145 prostate cancer cells (42). Compared with PC3 cells, emodin significantly induced the apoptosis of LNCaP cells and inhibited their proliferation. These findings suggested that emodin inhibits prostate $\mathrm{LNCaP}$ cell proliferation by regulating the activity of AR and p53-p21 pathways, increasing caspase -3 and -9 , increasing the ratio of $\mathrm{Bax} / \mathrm{Bcl}-2$, and inducing LNCaP cell apoptosis through mitochondrial signaling pathway (43). Emodin enhanced the cytotoxicity of chemotherapeutic drugs in prostate cancer cells, and its mechanism was found to be related to the inhibition of multidrug resistance (MDR) and hypoxia-inducing factors. Emodin is proven to be an ROS generator and a novel small inhibitor of HIF-1. It has been shown that co-treatment with emodin plus cisplatin in DU145 cells and mouse xenografts dramatically elevated ROS levels, downregulated MDR1 expression and HIF-1 transactivation. HIF1 acts as an upstream controller of MDR1 and is redox-sensitive (44).

Emodin was found to downregulate the transcriptional activity of AR by preventing AR nuclear translocation, disrupting the association between $\mathrm{AR}$ and heat shock protein 90 (HSP90), increasing the interaction and ubiquitination of AR with E3 ligase MDM2 (murine double minute 2), leading to AR degradation through a proteasome-mediated pathway in a ligand-independent manner (8). In in vivo studies, emodin demonstrated low drug toxicity and maintained physical activity in prostate cancer-induced C3(1)/SV40 transgenic mice (8).

The activation of p53 can induce the expression of p21. Emodin increased the expression of p53 and p21 in LNCaP cells, thus inducing significant apoptosis. LNCaP is an ARand LRP1-positive prostate cancer cell line, and has been found to be more susceptible to emodin than PC-3 cells, which are AR-negative LRP-positive prostate cancer cells. LRP1 and $\mathrm{AR}$ are expressed in prostate cancer and are upregulated in hypoxic conditions. In AR-positive LNCaP cells, AR was markedly upregulated under $\mathrm{CoCl}_{2}$-induced hypoxia-like conditions, decreased by emodin and $\mathrm{CoCl}_{2}$ co-treatment. 


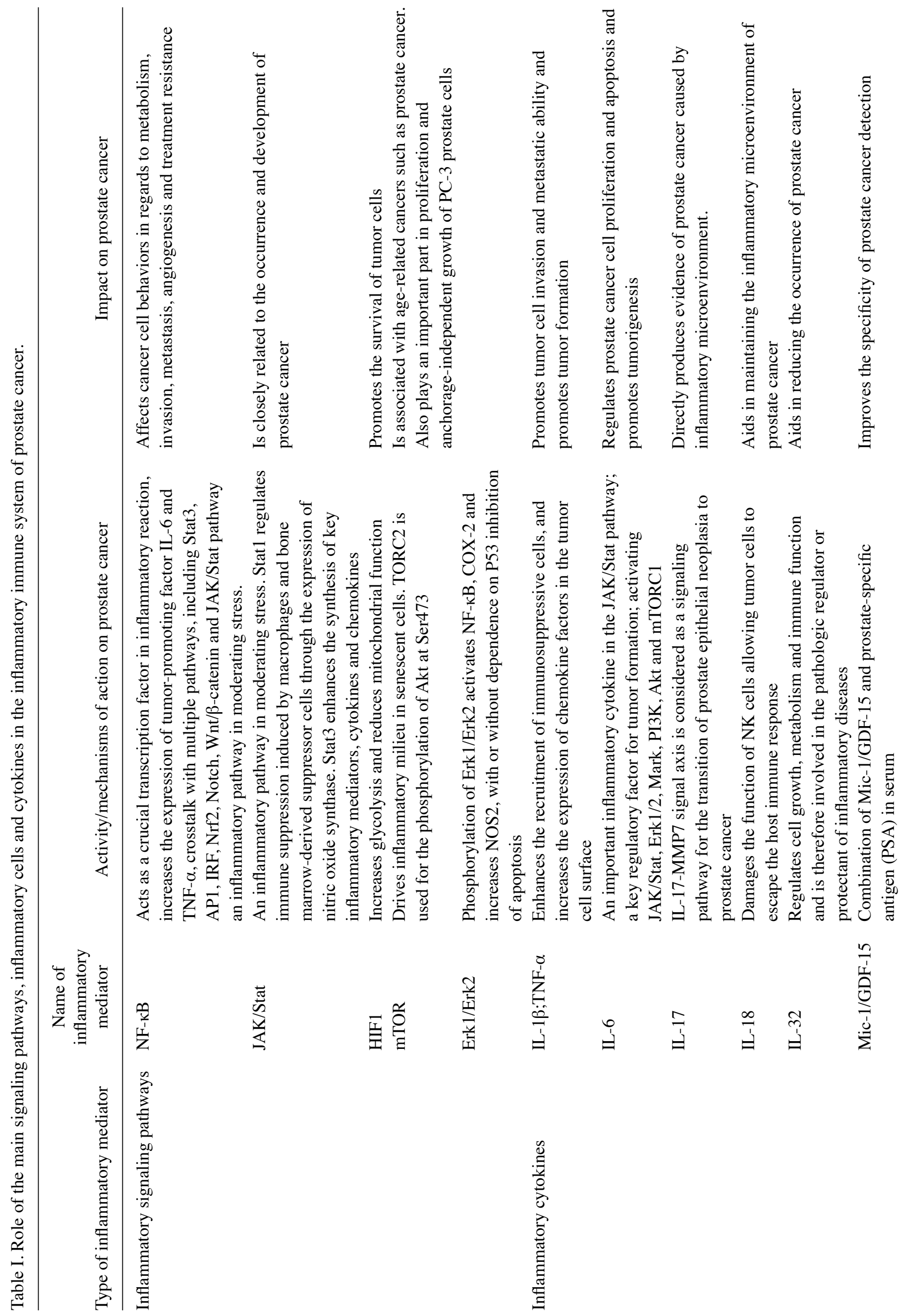




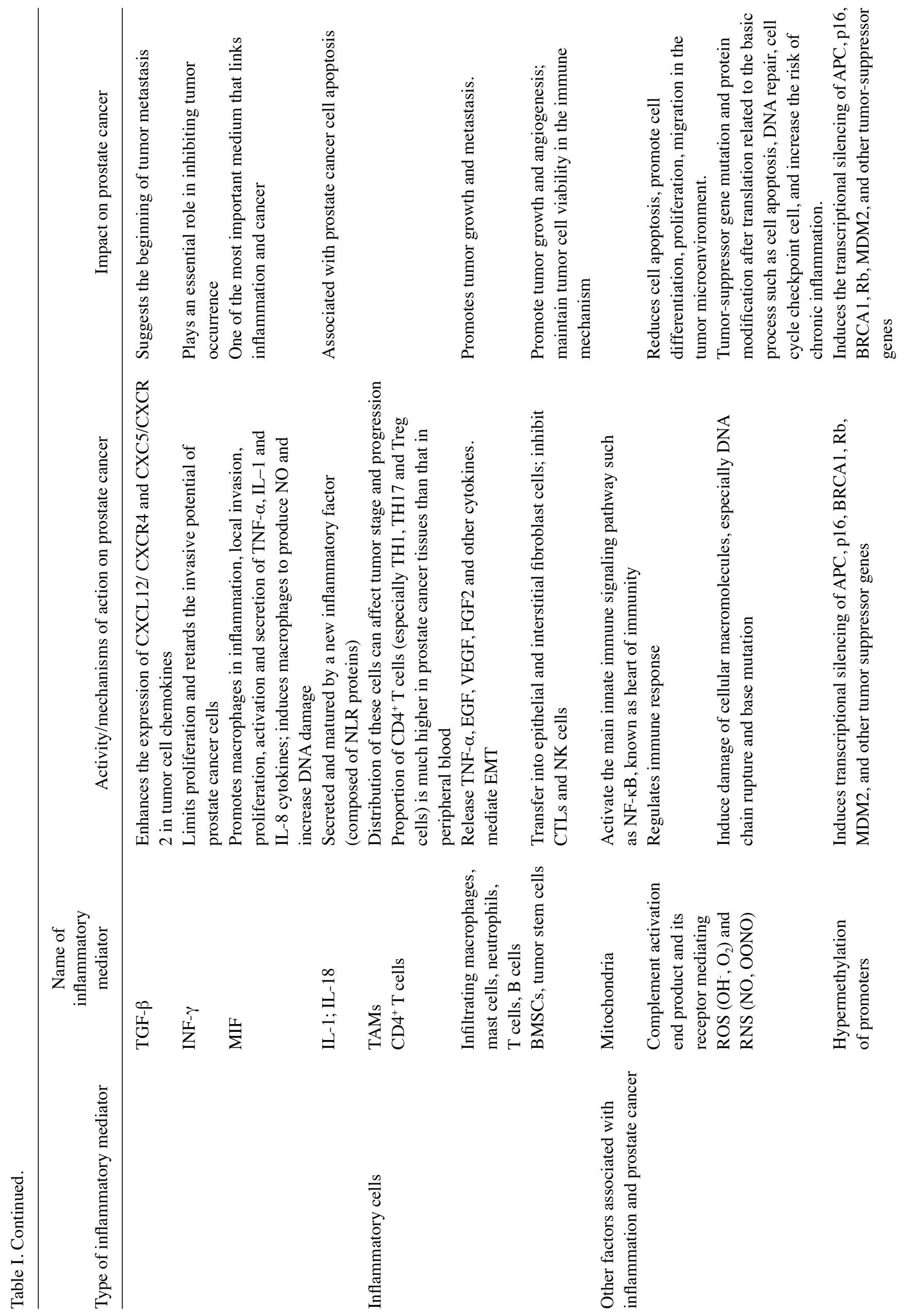




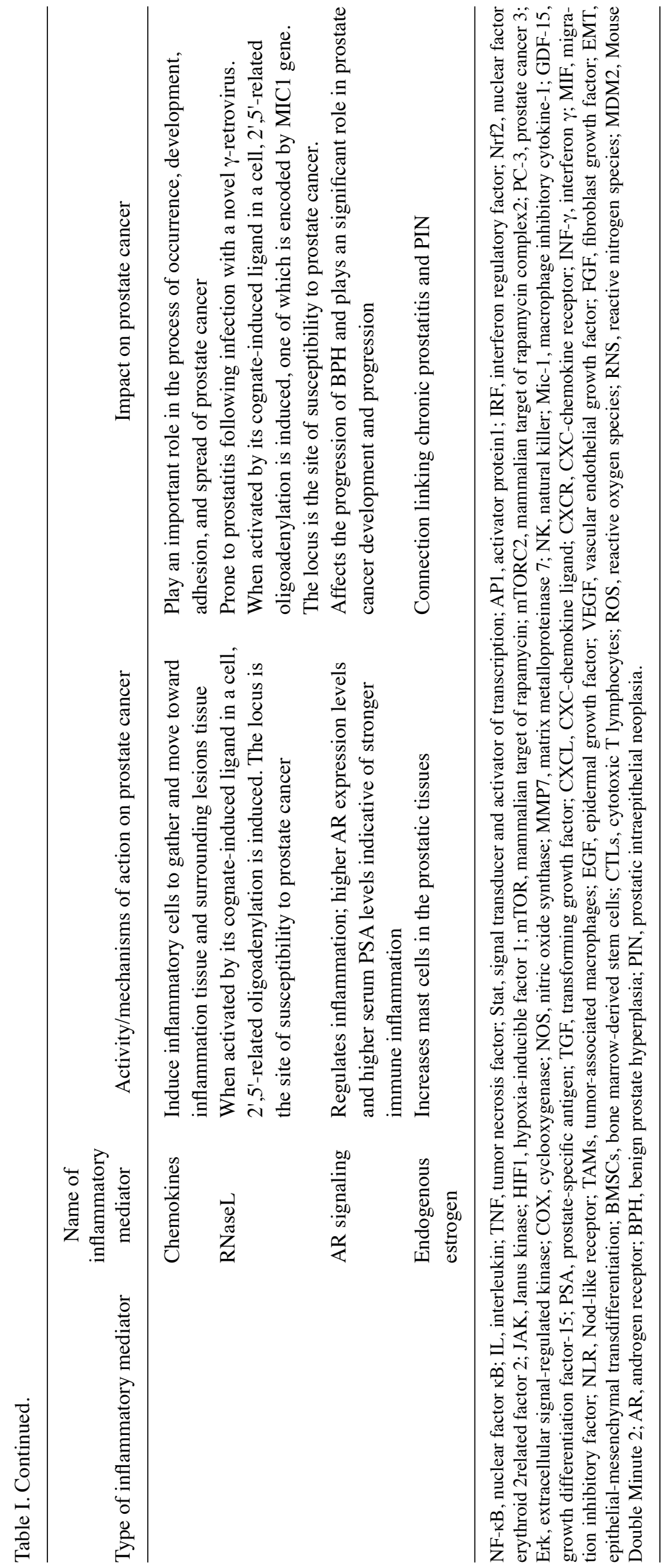


<smiles>CCOC(=O)c1cc(C)cc2c1C(=O)c1c(O)cc(O)cc1C2=O</smiles>

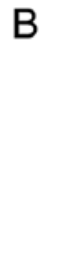

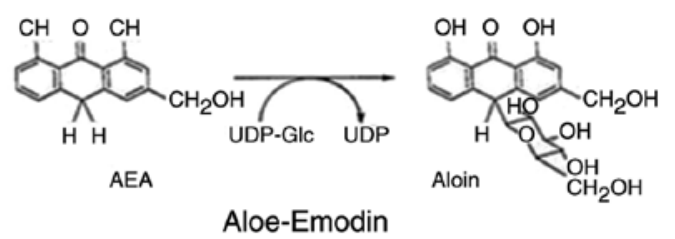<smiles>COc1cc(O)cc(C(=O)c2cc(C#N)cc(OC)c2C)c1</smiles>

D

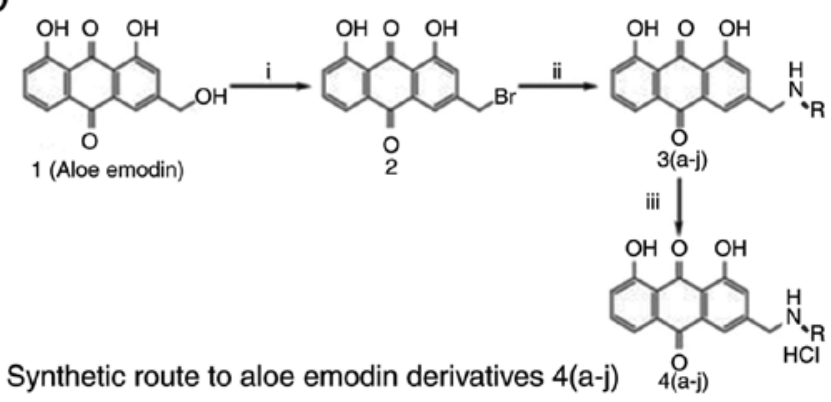

E

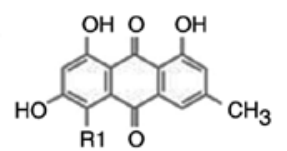

Substance: R1

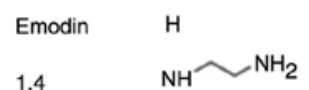

Emodin and

4-aminoethylamino-emodin

(L4)
$\mathrm{F} \mathrm{OHO} \mathrm{OH}$

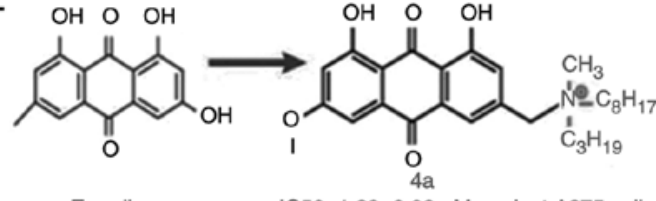

IC $50=1.39 \pm 0.02 \mu \mathrm{M}$ against $\mathrm{A} 375$ cells and lower toxicity in HELF cells

Quaternary ammonium salts of

Emodin (4a)
G<smiles>CC(C)Sc1cc2c(c3nsnc13)C(=O)c1ccccc1C2=O</smiles>

$6 \mathrm{~g}$ (NSC763968)

Sulfur-substituted anthra[1,2-c]

$[1,2,5]$ thiadiazole-6,11-diones $(6 \mathrm{~g})$

Figure 2. Chemical structure of emodin and some of its most active derivatives. (A) Emodin. (B) Aloe-Emodin. (C) Emodin-AMAD. (D) Synthetic route to aloe emodin derivatives 4(a-j). (E) Emodin and 4-aminoethylamino-emodin (L4). (F) Quaternary ammonium salts of Emodin(4a). (G) Sulfur-substituted anthra[1,2-c][1,2,5]thiadiazole-6,11-diones $(6 \mathrm{~g})$.

These data indicate that emodin induces ROS-mediated growth inhibition (41).

\section{Antitumor effects of emodin derivatives in prostate cancers}

Aloe vera is the most commercialized Aloe species belonging to the Xanthorrhoeaceae family (45). Aloe-Emodin (AE) (Fig. 2B) is an ahydroxyanthraquinone obtained from Aloe vera leaves, which is structurally very similar to emodin. AE was found to suppress the proliferation and anchorage-independent growth in PC-3 cells in a dose-dependent manner with a peak concentration at $15 \mathrm{mM}$. The phosphorylation of Akt at Ser473 was strongly inhibited by AE (15). In an ex vivo pull-down assay, AE-conjugated Sepharose 4B beads pulled down endogenous Rictor together with mTOR and Akt (15).

Emodin-derived aloin can combine with mTORC2 in the cells to inhibit kinase activity by inhibiting mTORC2, Akt and PKC- $\alpha$ substrate downstream activity. This in turn was found to inhibit the proliferation and nondependent growth of PC3 cells (15). This suggests that aloin can inhibit the development of prostate cancer through the mTOR signaling pathway (15) (Table II). An experiment that investigated the effect of AE in rat $\mathrm{C} 6$ glioma cells showed that $\mathrm{AE}$ led to the formation of intracytoplasmic acidic vesicles indicating autophagic cell death, blockage of the cell cycle and caspase-dependent apoptosis. AE had no affects on the activation of MAPK p38, Jun-N-terminal kinase, or transcription factor NF- $\mathrm{BB}$. But according to the results of cell-based ELISA, AE markedly inhibited the activation of extracellular signal-regulated kinases 1 and 2 (ERK1/2) in C6 cells. These results indicated that anti-glioma action of AE involved ERK-independent induction of both apoptosis and autophagy (46). However, whether AE also shows these effects in prostate cancer warrants further investigation.

AMAD (Fig. 2C), an emodin azide methyl anthraquinone derivative extracted from the nature knotweed rhizome, has potent cytotoxic effects on human breast cancer cell line MDA-MB-453 and human lung adenocarcinoma Calu-3 cells. Moreover, AMAD was found to induce apoptosis via a mitochondrial pathway involving caspase-8/Bid activation in both cell lines (47). Overexpression of HER2/neu is well-known to predict a poor prognosis in cancer. Another study showed that AMAD has the capability of potently decreasing Her2/neu protein and inhibiting the downstream MAPK and PI3K-Akt signaling pathways in dose- and time-dependent manners (48). Realistic data suggested that blockage of Her2/neu binding to Hsp90 followed by proteasomal degradation of Her $2 /$ neu were involved in emodin AMAD-induced apoptosis in Her2/neu-overexpressing cancer 
Table II. Mechanisms of emodin in prostate cancer.

\begin{tabular}{|c|c|c|}
\hline Natural drug name & Mechanism in prostate cancer & Impact on prostate cancer \\
\hline \multirow[t]{3}{*}{ Emodin } & Inhibits PIM kinase & Inhibits the growth of DU-145 prostate cancer cells \\
\hline & $\begin{array}{l}\text { Activates Notch signaling pathway; inhibits } \\
\text { VEGF }\end{array}$ & $\begin{array}{l}\text { Inhibited the growth of PC } 3 \text { prostate cancer cells, } \\
\text { induced cell apoptosis; and blocked the cell cycle in } \\
\text { G2/M phase; anti-cancer mechanisms }\end{array}$ \\
\hline & $\begin{array}{l}\text { Reduces the expression of AR in oxygen and } \\
\text { low-oxygen environment }\end{array}$ & Effect in LNCaP prostate cancer cells \\
\hline Emodin derivative aloin & $\begin{array}{l}\text { Inhibits mTOR signaling pathway through } \\
\text { inhibition of mTORC } 2 \text {, Akt and PKC- } \alpha \\
\text { substrate downstream activity }\end{array}$ & $\begin{array}{l}\text { Inhibits the proliferation and nondependent growth of } \\
\text { PC3 cells in the development of prostate cancer }\end{array}$ \\
\hline Emodin & $\begin{array}{l}\text { Inhibits the activation of NF- } \kappa \mathrm{B} \text { and lowers } \\
\text { CXCR } 4 \text { and HER } 2\end{array}$ & $\begin{array}{l}\text { Inhibit the invasion and metastasis of prostate cancer } \\
\text { cells }\end{array}$ \\
\hline Emodin & $\begin{array}{l}\text { Regulates the activity of AR and p53-p } 21 \\
\text { pathways, increases caspase- } 3 \text { and }-9 \text {, } \\
\text { increases the ratio of Bax/Bcl-2; and } \\
\text { induces LNCaP cell apoptosis through the } \\
\text { mitochondrial pathway }\end{array}$ & Inhibits prostate $\mathrm{LNCaP}$ cell proliferation \\
\hline Emodin & $\begin{array}{l}\text { Inhibits multidrug resistance and } \\
\text { hypoxia-inducing factors }\end{array}$ & $\begin{array}{l}\text { Enhances the cytotoxicity of chemotherapy drugs in } \\
\text { prostate cancer cells }\end{array}$ \\
\hline $\begin{array}{l}\text { Co-treatment with } \\
\text { emodin plus cisplatin }\end{array}$ & $\begin{array}{l}\text { Dramatically elevates ROS levels, } \\
\text { downregulates MDR } 1 \text { expression and } \\
\text { HIF-1 transactivation }\end{array}$ & $\begin{array}{l}\text { Inhibit the tumor growth in DU145 cells and mouse } \\
\text { xenografts, owing to oxidative stress and MDR1 } \\
\text { down-regulation within tumors }\end{array}$ \\
\hline Emodin & $\begin{array}{l}\text { Downregulates AR transcriptional activity } \\
\text { by preventing AR nuclear translocation, } \\
\text { disrupts the association between AR and } \\
\text { heat shock protein } 90 \text {, increasing the } \\
\text { interaction and ubiquitination of AR with } \\
\text { E3 ligase MDM2, leading to AR degradation } \\
\text { through proteasome-mediated pathway in a } \\
\text { ligand-independent manner }\end{array}$ & $\begin{array}{l}\text { Low drug toxicity; maintains physical activity in } \\
\text { prostate cancer-induced C3(1)/SV40 transgenic mice }\end{array}$ \\
\hline Emodin & $\begin{array}{l}\text { Increases the expression of p53 and p } 21 \text {; } \\
\text { thus, inhibits ROS-mediated growth in AR- } \\
\text { and LRP1-positive prostate cell line LNCaP }\end{array}$ & Induces significant apoptosis in LNCaP cells \\
\hline Aloe-Emodin & Inhibits phosphorylation of Akt at Ser 473 & $\begin{array}{l}\text { Suppresses proliferation and anchorage-independent } \\
\text { growth in PC-3 cells }\end{array}$ \\
\hline
\end{tabular}

cells (48). Further investigation is needed to ascertain whether AMAD has a role in this respect in prostate cancer.

\section{Emodin analog development in prostate cancer}

Emodin has some drawbacks as a therapeutic agent, such as low water solubility, low bioavailability after oral admission and relatively high toxicity to the liver and kidney (7). The structural modification of emodin side chain, such as polymethyleneamine, sugar, or minocycline, combined with methyl, hydroxyl and aryl ring sites showed enhanced antitumor efficacy (49). The other achieved derivatives through intercalating amino groups and glycosidic bonds displayed higher levels of antitumor activities (50). For example, addition of sugar chains in the emodin C3-hydroxyl site not only increased its solubility but also significantly improved its antitumor activity. Similarly, when the hydroxyl groups of C-1 and C-8 position of emodin were methylated, its anti-proliferative activity also showed improvement (51). EM-d-Rha is an anthracene L-rhamnopyranoside derivative of emodin by connecting L-rhamnopyranosides to a planar aromatic molecule, which has 10 -fold stronger antitumor activity and growth inhibitory effects. Introduction of one or two positively charged side chains in the tricyclic coplanar structure of the tricyclic compounds, such as anthranone compound anthracenedion, decreased the toxic effect on tumor cells (51). The 


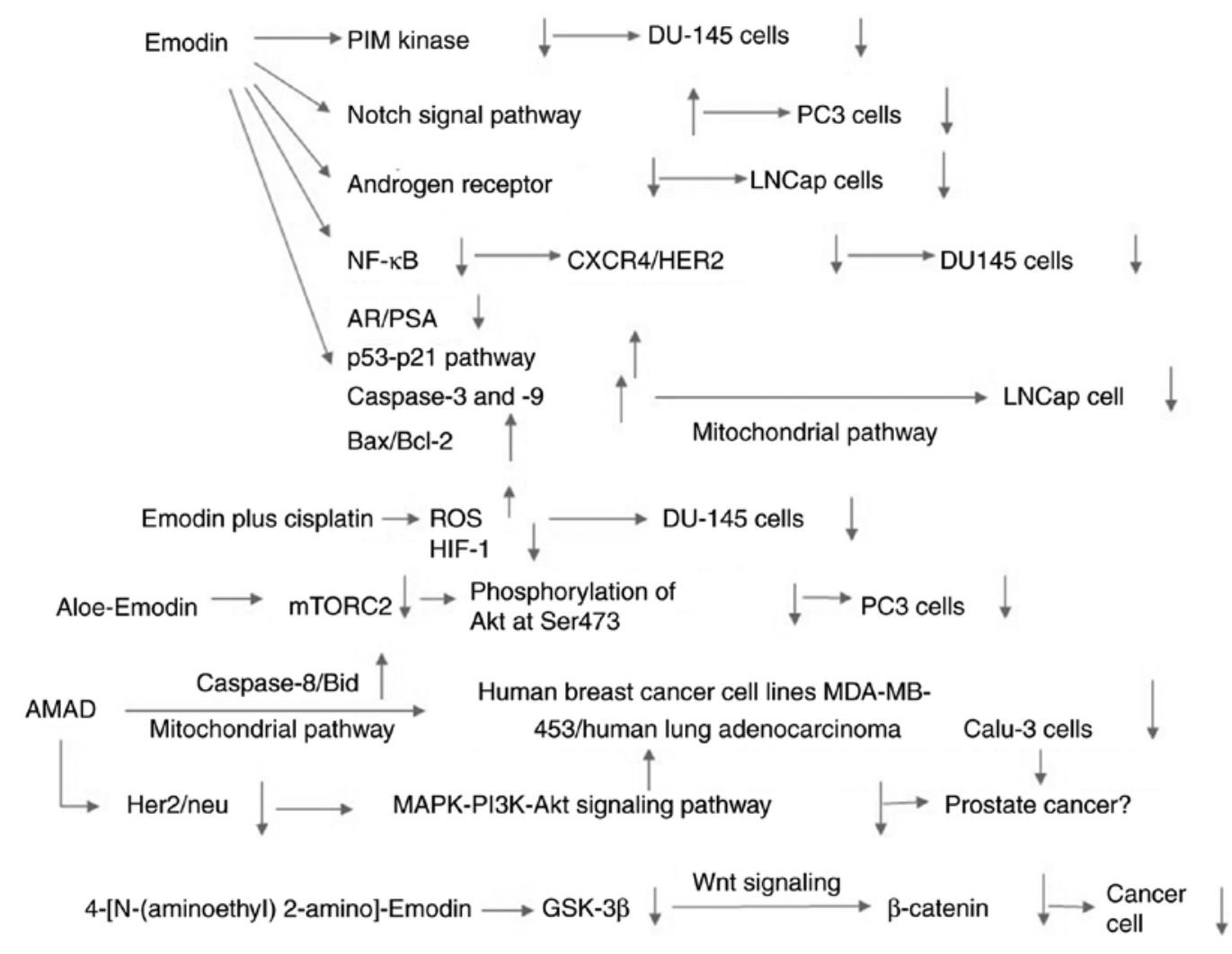

Figure 3. The signaling pathways with marked action of emodin (or derivatives) involved in prostate cancer development.

use of emodin for tumor treatment may be one of the future research directions if the side effects of emodin can be reduced without affecting its antitumor effect.

Through the combination of amino acid esters and substituted aromatic amines, water-soluble derivatives of AE have been synthesized (Fig. 2D). These compounds have demonstrated a more effective antitumor activity in HepG2 and NCI-H460 cells. The structural activity relationship of L-serine methyl ester, $\beta$-alanine ethyl ester and 3-(2-aminoethyl) pyridine substituents showed improvement in their antitumor activity (52). GSK-3 $\beta$ plays a vital role in Wnt signal transduction by mediating degradation of $\beta$-catenin. Revoking GSK-3 $\beta$-dependent phosphorylation of $\beta$-catenin by Wnt growth factors or inhibitors of GSK-3 $\beta$ led to the accumulation of hypophosphorylated uncomplexed $\beta$-catenin in the cytosol and nucleus, prompting the transcription of target gene. A new emodin derivative 4-[N-(aminoethyl) 2-amino]-Emodin (L4 compound) (Fig. 2E) acts as a strong GSK-3 $\beta$ inhibitor. It binds to the ATP binding site that is close to the two key residues Asp133 and Val135 and prevents TCF/LEF transactivation. Meanwhile, the L4 compound demonstrated low cytotoxicity when compared with other GSK-3 $\beta$ inhibitors (53).

A series of novel quaternary ammonium salts of emodin were synthesized in an experiment and their anticancer activities have been investigated in vitro in A375, BGC-823, HepG 2 and HELF cells. The results revealed that compound 4a (Fig. 2F) has the ability to induce morphological changes and decrease cell viability. Compound 4a induced apoptosis of A375 cells by dissipating mitochondrial membrane potential $(\Delta \Psi \mathrm{m})$, resulting in the upregulation of P53 and caspase-3. In addition, the results of this research group showed no direct correlation between alkylating reactivities of emodin derivatives and their anticancer activities and the presence of only a weak interaction between emodin and DNA. This implied that DNA might not be the main target of emodin derivatives. At the same time, the compound's hydrophilicity was unfavorable intracellularly. But a conclusion can be drawn that there is a close relationship between the ability to generate ROS and anticancer activity. Both compound $4 \mathrm{~d}$ and $4 \mathrm{a}$ were found to be fat soluble and contain one and two long carbon chains in $\mathrm{N}$ cations, respectively. Compound $4 \mathrm{a}$ has the highest capability to generate $\operatorname{ROS}$ ( $4 \mathrm{a}>4 \mathrm{~d}>$ emodin) (54). A series of sulfur-substituted anthra[1,2-c][1,2,5]thiadiazole-6,11-diones were synthesized. Among the tested compounds, 6g (Fig. 2G) appears to be the most active compound of this series that not only induced apoptosis in DU-145 cancer cells but also attenuated ERK1/2 and p38 signaling pathways. Further development of these compounds as potential anticancer agents is required (55).

\section{Prospects of emodin in the inflammatory immune microenvironment of prostate cancer}

There is limited research regarding the mechanism of emodin in the inflammatory immune microenvironment of prostate cancer, and the affect on the immune microenvironment in a tumor by this agent has raised wide concern $(56,57)$. The following suggestions have been proposed for future research.

The difference between beneficial inflammation that stimulates the body's positive immunity and detrimental inflammation that aggravates pathological damage must be explored. For example, the M1 phenotype can promote the 
differentiation of monocytes into macrophages and promote the sustained and effective adaptive immune response to tumors via the TLR4-Myd88 signaling pathway. In the pathogenesis of prostate cancer, how can benign immunity be triggered with agents is still an unanswered question. The exact mechanisms involved in transforming a tumor-promoted microenvironment (TH2 cells and M2 macrophages) to a tumor-inhibited microenvironment (TH1 cells and M1 macrophages) warrants further elucidation.

Sex steroid hormones act as influential factors in the occurrence, development, and outcomes of prostate cancer. One reason for this might be due to the innate immune system or the nonspecific immune system regulation of inflammation (58); macrophages in prostate cancer can produce IL-1, in which the selective androgen receptor modulators (SARMs) were used to activate the function of androgen receptor-induced gene instead of inhibition. This process contains TAB2 protein, which is a type of inflammatory signal sensor, and is the component of TAB2/N-CoR/HDAC blocker complex. Inflammation induces the phosphorylation of TAB2, triggering more gene transcription, and effecting prostate cancer by regulating the inflammatory microenvironment. The mechanism of emodin in hormone intervention treatment of prostate cancer should be further elucidated.

TAMs are specialized partners of tumor cell migration, invasion, and metastasis, and their affect on tumor cell metastasis is based on the macrophage environment. It has been reported that macrophages have increased peritoneal proliferation in ovarian cancer cells, and whether such a condition exists in prostate cancer is still unclear. ANXA5 usually induces anti-inflammatory and cell death activities. It has been found that ANXA5 plays an inhibitory role on Cox-2 in prostate cancer and could induce phosphorylation of $\mathrm{NF}-\kappa \mathrm{Bp} 65$, but its role in cancer is unclear $(13,59,60)$. Programmed death ligand 1 (PD-L1) interacts with programmed death 1 (PD-1) receptor on $\mathrm{T}$ cells to induce an immune response, which induces tumor cells to avoid immune monitoring. The role of anti-PD-1 antibodies and drugs in tumor immunotherapy has attracted much attention (61). It is interesting to understand how emodin regulates and interferes with PD-L1/PD-1, thereby affecting tumor immunity. Mesenchymal stem cells (MSCs) are a major concern in studying cancer and the immune microenvironment in recent years. MSCs are often recruited for local inflammation and tumor microenvironment, promoting the production of proinflammatory cytokines. The same is true for prostate cancer, and its specific mechanisms and drug intervention study should be further identified (3).

Abnormal expression of integrin induces tumor cell migration and invasion abilities, which can change the intracellular signal transduction to make them survive in the microenvironement of other organs and does not trigger the internal mechanisms of apoptosis (62). It can also cause drug resistance in tumor cells (63). The abnormal expression of $\alpha 6 \beta 1$ in prostate cancer can promote the metastasis of prostate cancer, activate $\mathrm{PI} 3 \mathrm{~K} / \mathrm{AKT}$ and $\mathrm{NF}-\kappa \mathrm{B}$ signaling pathways, and inhibit cell apoptosis, thus maintaining the survival of tumor cells. The role of emodin on this aspect should be further studied.

The acquisition and maintenance of type M1/M2 macrophage phenotypes depends on the regulation of transcription and post-transcriptional levels. Epigenetic changes are the key molecular mechanisms for controlling heterogeneity and plasticity of macrophages. Of these, the study on microRNAs (miRNAs), DNA methylation (DNAm), and epigenetic regulation of histone modification has been extensively studied. miRs are important regulatory factors in the proliferation, differentiation, and apoptosis of macrophage cells, and regulation of phenotypic balance of macrophages in miRs can alleviate inflammation and immune function $(64,65)$.

In conclusion, the inflammatory response should be considered as an increased risk of prostate cancer. Emodin exhibits anticancer action by targeting the inflammatory pathway such as ROS, HIF-1, PIM1, AR, p53 and PI3K-Akt-mTOR, providing a prospective area as an adjuvant therapy. Chemical modification may improve the solubility and utilization ratio of emodin avoiding the side effects; this issue deserves further investigation.

\section{Acknowledgements}

YT thanks Professor Benyi Li, who is from the Department of Urology, the University of Kansas Medical Center, Kansas City, USA. He helped to revise and correct the article. YT thanks Professor Zhaoqin Fang and Professor Aidong Yang for their continued encouragement and support. With their support, YT was able to complete her thesis successfully.

\section{Funding}

The present study was supported partially by grants from National Natural Science Foundation of China (grant no. 81673855), the Heritage Talent Project of Shanghai University of Traditional Chinese Medicine of China (grant no. A1-GY010101), and partially by the Cinical Basic Subject of Shanghai University of Traditional Chinese Medicine of China (grant no. 12ZLJ08).

\section{Availability of data and materials}

Not applicable.

\section{Authors' contributions}

YT conceived the research design, collected the literature, researched the information, constructed the figures and table and wrote the paper. ZF, AY, BT and ZW carried out the literature analysis, revised the paper and provided funding support. All authors reviewed the results and approved the final version of the manuscript.

\section{Ethics approval and consent to participate}

Not applicable.

\section{Patient consent for publication}

Not applicable.

\section{Competing interests}

The authors declare that they have no competing interests. 


\section{References}

1. Torre LA, Siegel RL, Ward EM and Jemal A: Global cancer incidence and mortality rates and trends-an update. Cancer Epidemiol Biomarkers Prev 25: 16-27, 2016.

2. Pang C, Guan Y, Li H, Chen W and Zhu G: Urologic cancer in China. Jpn J Clin Oncol 46: 497-501, 2016.

3. Yang KQ, Liu Y, Huang QH, Mo N, Zhang QY, Meng QG and Cheng JW: Bone marrow-derived mesenchymal stem cells induced by inflammatory cytokines produce angiogenetic factors and promote prostate cancer growth. BMC Cancer 17: 878, 2017.

4. Balkwill $\mathrm{F}$ and Mantovani A: Inflammation and cancer: Back to virchow? Lancet 357: 539-545, 2001

5. Daniels NA, Ewing SK, Zmuda JM, Wilt TJ and Bauer DC; Osteoporotic Fractures in Men (MrOS) Research Group: Correlates and prevalence of prostatitis in a large community-based cohort of older men. Urology 66: 964-970, 2005.

6. De Marzo AM, Marchi VL, Epstein JI and Nelson WG: Proliferative inflammatory atrophy of the prostate: Implications for prostatic carcinogenesis. Am J Pathol 155: 1985-1992, 1999.

7. Dong X, Fu J, Yin X, Cao S, Li X, Lin L and Ni J; Huyiligeqi: Emodin: A review of its pharmacology, toxicity and pharmacokinetics. Phytother Res 30: 1207-1218, 2016.

8. Cha TL, Qiu L, Chen CT, Wen Y and Hung MC: Emodin down-regulates androgen receptor and inhibits prostate cancer cell growth. Cancer Res 65: 2287-2295, 2005.

9. Diakos CI, Charles KA, McMillan DC and Clarke SJ: Cancer-related inflammation and treatment effectiveness. Lancet Oncol 15: e493-e503, 2014

10. Liu Z, Xiao B, Mao XH and Zou QM: Research progress on relationship between inflammationand tumor. Prog Mod Biomed 9: 591-594, 2009.

11. Karan D and Dubey S: From inflammation to prostate cancer: The role of inflammasomes. Adv Urol 2016: 3140372, 2016.

12. Taniguchi $\mathrm{K}$ and Karin M: NF- $\kappa \mathrm{B}$, inflammation, immunity and cancer: Coming of age. Nat Rev Immunol 18: 309-324, 2018.

13. Mantovani A, Allavena P, Sica A and Balkwill F: Cancer-related inflammation. Nature 454: 436-444, 2008.

14. Laberge RM, Sun Y, Orjalo AV, Patil CK, Freund A, Zhou L, Curran SC, Davalos AR, Wilson-Edell KA, Liu S, et al: MTOR regulates the pro-tumorigenic senescence-associated secretory phenotype by promoting IL1A translation. Nat Cell Biol 17: 1049-1061, 2015.

15. Liu K, Park C, Li S, Lee KW, Liu H, He L, Soung NK, Ahn JS, Bode AM, Dong Z, et al: Aloe-emodin suppresses prostate cancer by targeting the mTOR complex 2 . Carcinogenesis 33 : 1406-1411, 2012

16. Denko NC: Hypoxia, HIF1 and glucose metabolism in the solid tumour. Nat Rev Cancer 8: 705-713, 2008.

17. Shalapour S and Karin M: Immunity, inflammation, and cancer: An eternal fight between good and evil. J Clin Invest 125: 3347-3355, 2015 .

18. Zhang Y, Liang C and Chen X: Research progress on the relationship between chronic prostatic inflammation and prostate cancer. J Mod Urol 20: 207-210, 2015.

19. Zhang Q, Liu S, Ge D, Zhang Q, Xue Y, Xiong Z, Abdel-Mageed AB, Myers L, Hill SM, Rowan BG, et al: Interleukin-17 promotes formation and growth of prostate adenocarcinoma in mouse models. Cancer Res 72: 2589-2599, 2012.

20. Hong JT, Son DJ, Lee CK, Yoon DY, Lee DH and Park MH: Interleukin 32, inflammation and cancer. Pharmacol Ther 174 127-137, 2017.

21. Wang Z and Qi Y: Inflammation: Tumor catalyst. World Latest Med Inf 16: 70-71, 2016.

22. Sfanos KS, Yegnasubramanian S, Nelson WG and De Marzo AM: The inflammatory microenvironment and microbiome in prostate cancer development. Nat Rev Urol 15: 11-24, 2018.

23. Sfanos KS, Bruno TC, Maris $\mathrm{CH}, \mathrm{Xu} \mathrm{L}$, Thoburn CJ, DeMarzo AM, Meeker AK, Isaacs WB and Drake CG: Phenotypic analysis of prostate-infiltrating lymphocytes reveals TH17 and Treg skewing. Clin Cancer Res 14: 3254-3261, 2008.

24. Su huan and Chen ming: Research progress on the mechanism of inflammatory response and tumor microenvironment in prostate cancer. J Southeast Univ 36: 847-851, 2017 (Medical Science Edition).

25. Mills EL, Kelly B and O'Neill LAJ: Mitochondria are the powerhouses of immunity. Nat Immunol 18: 488-498, 2017.

26. Afshar-Kharghan V: The role of the complement system in cancer. J Clin Invest 127: 780-789, 2017.
27. Zhou W, Hu W and Xu W: Effects of CXCL16/CXCR6 axis on proliferation and invasion of human prostate cancer cell line in vitro. Med J Wuhan University 31: 479-482, 2010.

28. Kuo PL, Shen KH, Hung SH and Hsu YL: CXCL1/GROa increases cell migration and invasion of prostate cancer by decreasing fibulin-1 expression through NF- $\mathrm{BB} / \mathrm{HDAC} 1$ epigenetic regulation. Carcinogenesis 33: 2477-2487, 2012.

29. Yang L, et al: The relationship between chemokines, inflammation, and prostate cancer. Mod Prev Med 42: 952-956, 2015.

30. Schoenfeld JD, Margalit DN, Kasperzyk JL, Shui IM, Rider JR, Epstein MM, Meisner A, Kenfield SA, Martin NE, Nguyen PL, et al: A single nucleotide polymorphism in inflammatory gene RNASEL predicts outcome after radiation therapy for localized prostate cancer. Clin Cancer Res 19: 1612-1619, 2013.

31. Wiklund F, Jonsson BA, Brookes AJ, Strömqvist L, Adolfsson J, Emanuelsson M, Adami HO, Augustsson-Bälter K and Grönberg H: Genetic analysis of the RNASEL gene in hereditary, familial, and sporadic prostate cancer. Clin Cancer Res 10: 7150-7156, 2004

32. Izumi K, Li L and Chang C: Androgen receptor and immune inflammation in benign prostatic hyperplasia and prostate cancer. Clin Investig (Lond) 4: 935-950, 2014.

33. Wu ZL, Yuan Y, Geng H and Xia SJ: Influence of immune inflammation on androgen receptor expression in benign prostatic hyperplasia tissue. Asian J Androl 14: 316-319, 2012.

34. Wang X, Lin WJ, Izumi K, Jiang Q, Lai KP, Xu D, Fang LY, Lu T, Li L, Xia S and Chang C: Increased infiltrated macrophages in benign prostatic hyperplasia $(\mathrm{BPH})$ : Role of stromal androgen receptor in macrophage-induced prostate stromal cell proliferation. J Biol Chem 287: 18376-18385, 2012.

35. Vignozzi L, Cellai I, Santi R, Lombardelli L, Morelli A, Comeglio P, Filippi S, Logiodice F, Carini M, Nesi G, et al: Antiinflammatory effect of androgen receptor activation in human benign prostatic hyperplasia cells. J Endocrinol 214: 31-43, 2012.

36. Ellem SJ, Wang H, Poutanen M and Risbridger GP: Increased endogenous estrogen synthesis leads to the sequential induction of prostatic inflammation (prostatitis) and prostatic pre-malignancy. Am J Pathol 175: 1187-1199, 2009.

37. Aryee MJ, Liu W, Engelmann JC, Nuhn P, Gurel M, Haffner MC, Esopi D, Irizarry RA, Getzenberg RH, Nelson WG, et al: DNA methylation alterations exhibit intraindividual stability and interindividual heterogeneity in prostate cancer metastases. Sci Transl Med 5: 169ra10, 2013.

38. Mani RS, Amin MA, Li X, Kalyana-Sundaram S, Veeneman BA, Wang L, Ghosh A, Aslam A, Ramanand SG, Rabquer BJ, et al: Inflammation-induced oxidative stress mediates gene fusion formation in prostate cancer. Cell Rep 17: 2620-2631, 2016.

39. Giraud F, Akué-Gédu R, Nauton L, Candelon N, Debiton E, Théry V, Anizon F and Moreau P: Synthesis and biological activities of 4-substituted pyrrolo[2,3-a]carbazole Pim kinase inhibitors. Eur J Med Chem 56: 225-236, 2012.

40. Deng G, Ju X, Meng Q, Yu ZJ and Ma LB: Emodin inhibits the proliferation of PC3 prostate cancer cells in vitro via the Notch signaling pathway. Mol Med Rep 12: 4427-4433, 2015.

41. Masaldan S and Iyer VV: Exploration of effects of emodin in selected cancer cell lines: Enhanced growth inhibition by ascorbic acid and regulation of LRP1 and AR under hypoxia-like conditions. J Appl Toxicol 34: 95-104, 2014.

42. Ok S, Kim SM, Kim C, Nam D, Shim BS, Kim SH, Ahn KS Choi SH and Ahn KS: Emodin inhibits invasion and migration of prostate and lung cancer cells by downregulating the expression of chemokine receptor CXCR4. Immunopharmacol Immunotoxicol 34: 768-778, 2012.

43. Yu CX, Zhang XQ, Kang LD, Zhang PJ, Chen WW, Liu WW, Liu QW and Zhang JY: Emodin induces apoptosis in human prostate cancer cell LNCaP. Asian J Androl 10: 625-634, 2008.

44. Huang XZ, Wang J, Huang C, Chen YY, Shi GY, Hu QS and Yi J: Emodin enhances cytotoxicity of chemotherapeutic drugs in prostate cancer cells: The mechanisms involve ROS-mediated suppression of multidrug resistance and hypoxia inducible factor-1. Cancer Biol Ther 7: 468-475, 2008.

45. Kumar S, Yadav M, Yadav A, Rohilla P and Yadav JP. Antiplasmodial potential and quantification of aloin and aloe-emodin in Aloe vera collected from different climatic regions of India. BMC Complement Altern Med 17: 369, 2017.

46. Mijatovic S, Maksimovic-Ivanic D, Radovic J, Miljkovic DJ, Harhaji LJ, Vuckovic O, Stosic-Grujicic S, Mostarica Stojkovic M and Trajkovic V: Anti-glioma action of aloe emodin: The role of ERK inhibition. Cell Mol Life Sci 62: 589-598, 2005. 
47. Yan Y, Su X, Liang Y, Zhang J, Shi C, Lu Y, Gu L and Fu L: Emodin azide methyl anthraquinone derivative triggers mitochondrial-dependent cell apoptosis involving in caspase-8mediated bid cleavage. Mol Cancer Ther 7: 1688-1697, 2008.

48. Yan YY, Zheng LS, Zhang X, Chen LK, Singh S, Wang F, Zhang JY, Liang YJ, Dai CL, Gu LQ, et al: Blockade of Her2/neu binding to Hsp90 by emodin azide methyl anthraquinone derivative induces proteasomal degradation of Her2/neu. Mol Pharm 8 : $1687-1697,2011$

49. Yan YY, Fu LW, Zhang W, Ma HS, Ma CG, Liang YJ, Liu BY, Yu JZ, Wu QZ and Dong YM:Emodin azide methyl anthraquinone derivative induced G0/G1 arrest in HER2/neu-overexpressing MDA-MB-453 breast cancer cells. J BUON 19: 650-655, 2014.

50. Wen-Feng W, Feng-Sen Z, Wen-Na Z, Ze-Dong B, Hui-Jun Y, Jing-Wei S and Yao-Feng Y: The synthesis, structural study and anticancer activity evaluation of emodin derivatives containing conjugative groups. Med Chem 9: 545-552, 2013.

51. Xing JY, Song GP, Deng JP, Jiang LZ, Xiong P, Yang BJ and Liu SS: Antitumor effects and mechanism of novel emodin rhamnoside derivatives against human cancer cells in vitro. PLoS One 10: e0144781, 2015.

52. Thimmegowda NR, Park C, Shwetha B, Sakchaisri K, Liu K, Hwang J, Lee S, Jeong SJ, Soung NK, Jang JH, et al: Synthesis and antitumor activity of natural compound aloe emodin derivatives. Chem Biol Drug Des 85: 638-644, 2015.

53. Gebhardt R, Lerche KS, Götschel F, Günther R, Kolander J, Teich L, Zellmer S, Hofmann HJ, Eger K, Hecht A and Gaunitz F: 4-Aminoethylamino-emodin-a novel potent inhibitor of GSK-3beta-acts as an insulin-sensitizer avoiding downstream effects of activated beta-catenin. J Cell Mol Med 14: 1276-1293, 2010.

54. Yang X, Zhao W, Hu X, Hao X, Hong F, Wang J, Xiang L, Zhu Y, Yuan Y, Ho RJ, et al: Synthesis, characterization, and anticancer activity of novel lipophilic emodin cationic derivatives. Chem Biol Drug Des 86: 1451-1457, 2015.

55. Lee YR, Chen TC, Lee CC, Chen CL, Ahmed Ali AA, Tikhomirov A, Guh JH, Yu DS and Huang HS: Ring fusion strategy for synthesis and lead optimization of sulfur-substituted anthra[1,2-c][1,2,5]thiadiazole-6,11-dione derivatives as promising scaffold of antitumor agents. Eur J Med Chem 102: 661-676, 2015.
56. Silva JAF, Bruni-Cardoso A, Augusto TM, Damas-Souza DM, Barbosa GO, Felisbino SL, Stach-Machado DR and Carvalho HF: Macrophage roles in the clearance of apoptotic cells and control of inflammation in the prostate gland after castration. Prostate 78: 95-103, 2018

57. Dart DA, Uysal-Onganer P and Jiang WG: Prostate-specific PTen deletion in mice activates inflammatory microRNA expression pathways in the epithelium early in hyperplasia development. Oncogenesis 6: 400, 2017.

58. Mantovani A: Cancer: An infernal triangle. Nature 448: 547-548, 2007.

59. Baek HS, Park N, Kwon YJ, Ye DJ, Shin S and Chun YJ: Annexin A5 suppresses cyclooxygenase-2 expression by downregulating the protein kinase $\mathrm{C}-\zeta$-nuclear factor- $\kappa \mathrm{B}$ signaling pathway in prostate cancer cells. Oncotarget 8: 74263-74275, 2017.

60. Zhu P, Baek SH, Bourk EM, Ohgi KA, Garcia-Bassets I, Sanjo H, Akira S, Kotol PF, Glass CK, Rosenfeld MG and Rose DW: Macrophage/cancer cell interactions mediate hormone resistance by a nuclear receptor derepression pathway. Cell 124: 615-629, 2006.

61. Chen G, Huang AC, Zhang W, Zhang G, Wu M, Xu W, Yu Z, Yang J, Wang B, Sun H, et al: Exosomal PD-L1 contributes to immunosuppression and is associated with anti-PD-1 response. Nature 560: 382-386, 2018.

62. Winograd-Katz SE, Fässler R, Geiger B and Legate KR: The integrin adhesome: From genes and proteins to human disease. Nat Rev Mol Cell Biol 15: 273-288, 2014.

63. Eke I, Dickreuter E and Cordes N: Enhanced radiosensitivity of head and neck squamous cell carcinoma cells by $\beta 1$ integrin inhibition. Radiother Oncol 104: 235-242, 2012.

64. Wu XQ, Dai Y, Yang Y, Huang C, Meng XM, Wu BM and Li J: Emerging role of microRNAs in regulating macrophage activation and polarization in immune response and inflammation. Immunology 148: 237-248, 2016.

65. Ouimet M, Ediriweera HN, Gundra UM, Sheedy FJ, Ramkhelawon B, Hutchison SB, Rinehold K, van Solingen C, Fullerton MD, Cecchini K, et al: MicroRNA-33-dependent regulation of macrophage metabolism directs immune cell polarization in atherosclerosis. J Clin Invest 125: 4334-4348, 2015. 